Evaluation of the RADCHEM Diagnostic as an Assessment of Fuel-Ablator Mix and Fuel Rho R

D. A. Shaughnessy, C. Cerjan, K. J. Moody, L. Bernstein, R. Hoffman, M. A. Stoyer, R. Fortner, D. Schneider

March 9, 2011 
This document was prepared as an account of work sponsored by an agency of the United States government. Neither the United States government nor Lawrence Livermore National Security, LLC, nor any of their employees makes any warranty, expressed or implied, or assumes any legal liability or responsibility for the accuracy, completeness, or usefulness of any information, apparatus, product, or process disclosed, or represents that its use would not infringe privately owned rights. Reference herein to any specific commercial product, process, or service by trade name, trademark, manufacturer, or otherwise does not necessarily constitute or imply its endorsement, recommendation, or favoring by the United States government or Lawrence Livermore National Security, LLC. The views and opinions of authors expressed herein do not necessarily state or reflect those of the United States government or Lawrence Livermore National Security, LLC, and shall not be used for advertising or product endorsement purposes.

This work performed under the auspices of the U.S. Department of Energy by Lawrence Livermore National Laboratory under Contract DE-AC52-07NA27344. 


\title{
Evaluation of the RADCHEM Diagnostic as an Assessment of Fuel-Ablator Mix and Fuel Rho R
}

\author{
D.A. Shaughnessy, C. Cerjan, K.J. Moody, L. Bernstein, R. Hoffman, M.A. Stoyer, R. Fortner, and D. \\ Schneider \\ Lawrence Livermore National Laboratory, Livermore, CA, 94551, USA
}

\begin{abstract}
:
The RADCHEM diagnostic consists of the collection of both gaseous and solid debris samples following a NIF shot. Small amounts of detector elements are doped into the inner-most layer of the ablator shell, which then undergo nuclear activations with neutrons and/or charged particles that are produced during the fusion process. Reaction products are collected and their radioactive decays are counted in order to determine the number and type of activations that occurred. Simulations have shown that these data can then be correlated to several capsule parameters, including fuel rhoR ( $\rho R)$, implosion asymmetry, and fuel-ablator mix. In this document we present a brief summary of the simulations that have been performed, and evaluate the ability of RADCHEM to assess capsule performance. A longer, follow-on document, which will include more details on the specific simulations and experimental techniques that have been considered for RADCHEM, is currently being written.
\end{abstract}

\section{Introduction to the RADCHEM diagnostic}

The RADCHEM diagnostic consists of two systems, one for the collection of gases produced during a NIF shot with the Radiochemical Apparatus for Gaseous Samples (RAGS) instrument, and one for the collection of solid debris samples with a collector that will be inserted into the chamber before a shot and extracted immediately following the shot for analysis (currently being designed). Utilization of either system requires the NIF capsule to have a small amount of a "detector" element(s) loaded into the inner-most layer of the ablator shell (either plastic or Be) either in the form of a separated isotope, or generated in situ prior to fielding the capsule via neutron or charged particle irradiation. A "detector" is an isotope of an element that will undergo nuclear reactions with the neutrons and/or charged particles that are produced during the DT fusion process, but is not normally found in a NIF capsule. Simulations performed by $\mathrm{C}$. Cerjan have shown that the implosion performance of the capsule will not be jeopardized if $\leq 10^{15}$ detector element atoms are introduced into the ablator via ion implantation, sputtering, or similar method. The doped atoms should be located as close to the fuel as possible, and the initial number of atoms should be known before the capsule is shot in NIF, particularly when the element's atomic number changes, as in a charged particle reaction. A discussion of the fabrication of the RADCHEM capsule is beyond the scope of this document.

For neutron-induced reactions the amount of radiochemical detector activations produced is sensitive to the mass and spatial distribution of the loaded material during capsule burn. As a result, neutron-induced reactions are sensitive to rhoR $(\rho R)$, burn asymmetry, and mix. Detector elements do 
not change their atomic numbers as a result of neutron activation; noble gas detectors loaded into capsule ablator shells can therefore be collected with the RAGS apparatus with a reasonably high efficiency and subsequently measured from the collected debris material. A big advantage that the RADCHEM gas collection diagnostic has is that we can observe two nuclear reactions simultaneously that each have a different sensitivity to neutron energies, for example, ${ }^{124} \mathrm{Xe}(n, \gamma){ }^{125} \mathrm{Xe}$ and

${ }^{124} \mathrm{Xe}(n, 2 n){ }^{123} \mathrm{Xe}$. This enables us to break the dependence of the data on rhoR, mix and asymmetry and separate out just one of these variables, namely fuel areal density $(\rho R)$. In general, the $(n, \gamma)$ cross section is most sensitive to neutrons below $8 \mathrm{MeV}$ and the cross section increases as the neutron energy decreases. On the other hand, the $(n, 2 n)$ reaction is dominated by $14 \mathrm{MeV}$ neutrons. By measuring the ratio of the concentrations of these two radioactive products (i.e., ${ }^{125} \mathrm{Xe} /{ }^{123} \mathrm{Xe}$ ) we can determine the average energy of the downscattered neutrons and correlate this to the $\rho R$ of the cold fuel. Further analysis as to the value of each of the individual radioactive products (i.e., the absolute number of atoms produced in either the $(n, \gamma)$ or $(n, 2 n)$ reaction as opposed to their ratio) to infer information on mix and asymmetry is future work that needs to be done.

Beside neutrons there are charged particle-induced reactions that can also be used as diagnostics. First-order charged-particles produced via the DT-fusion process are limited to alpha particles, which are produced with $3.52 \mathrm{MeV}$ of energy directly in the burning hot spot. Our analysis will consist primarily of $(\alpha, n)$ reactions. One such reaction would be the ${ }^{18} \mathrm{O}(\alpha, n)^{21} \mathrm{Ne}$ reaction. While our analysis shows this would be a viable mix diagnostic, the difficulty is that ${ }^{21} \mathrm{Ne}$ is not radioactive and is also present as part of the chamber background. Further work to either reduce backgrounds or find other practical alpha reaction products is expected to be pursued, and the composition of the NIF target chamber background gases has been measured by C. Velsko (results are in progress.) At high yields where the signal is likely to overwhelm any contributions from background this could be an appropriate mix diagnostic. Second-order charged particle-induced reactions occur when neutrons interact with the dense, cold fuel and produce energetic particles comprised of fuel components ( $D, T, p)$. These chargedparticles have a limited range and are thus sensitive to the proximity of loaded detectors. Since these reactions are second-order, a significant capsule yield is required to make detectable amounts of product. For noble gases, the most promising second-order mix reaction is ${ }^{127} \mathrm{I}(d, 2 n)^{127} \mathrm{Xe}$, since we will be determining collection efficiency of xenon as part of the neutron-induced reactions described above. Other reactions will also be pursued in future work.

The primary advantage for neutron-induced reactions is they are "self-tracing," i.e., the collection efficiency is determined by measuring a ratio of reaction products that have the same atomic number. In this way, collection efficiency is removed as well as the need to know the absolute amount of xenon loaded, and the ratio itself is used to benchmark capsule performance. As discussed in Section II below, the quantity ${ }^{125} \mathrm{Xe} /{ }^{123} \mathrm{Xe}$ can be related to fuel $\rho \mathrm{R}$ without determining the absolute concentration of atoms or collection efficiency. This will be the preferred method for evaluating neutron-induced reactions. For these reactions we can also measure the number of unreacted ${ }^{124} \mathrm{Xe}$ atoms collected using noble gas mass spectrometry (NGMS) so we can determine the absolute collection efficiency as well. This will allow for further evaluation of possible mix indicators using single isotope measurements. For charged-particle reactions we need to know the initial amount of detector material 
loaded as well as the collection efficiency of the products. For the first studies of charged-particle reactions at NIF we have chosen Xe as the product to be collected since we will measure the xenon collection efficiency through our work with neutron-induced reactions. ${ }^{127}$ I will be loaded in a capsule to measure mix through the ${ }^{127} \mathrm{I}(d, 2 n)^{127}$ Xe reaction (discussed in more detail in Section III). Since iodine and xenon have very different chemical behavior, one must determine how much iodine was actually loaded before the shot. The process of mono-layer deposition, which is being proposed, should have fairly reproducible numbers for the number of ${ }^{127}$ I atoms loaded, but that needs to be confirmed experimentally. This strategy will be applied for most mix measurements where the final product is the result of a charged-particle reaction that has a different atomic number than the initial species.

Reactions that produce elements other than xenon will require measurement of the collection efficiency for the reaction products, which can only be accomplished using tracer isotopes. The tracer element must have the same atomic number as the final product of the detector element activation and be colocated in the ablator shell. The number of tracer atoms collected can be used to determine collection efficiency of the activated species.

In most of the simulations performed thus far, the detector elements were assumed to be loaded uniformly throughout the inner-most layer of the ablator except for the case of static hot spot mix in which the detector elements were uniformly distributed in the burning region. We have not yet determined the effect of asymmetric loadings, i.e., loading different elements in different halves of the capsule. Potential effects from geometric and chemical fractionation during the capsule explosion are currently unknown. However, several elements could simultaneously be loaded into the ablator shell in an attempt to measure both $\rho R$ and mix during a shot, as long as the total number of doped atoms was not large enough to affect the performance of the capsule. This will largely depend on capsule fabrication methods, and each product must either have a corresponding tracer or be self-traced through neutron reactions. These methods are worth investigating in the future, since RADCHEM diagnostics are an excellent way to evaluate what is happening deep within the capsule as the fusion process occurs.

It should be noted that the result from any RADCHEM measurement is either a total concentration of atoms or a ratio of isotopes. In either case the results must be subsequently interpreted using explosion code calculations in order to turn them into diagnostically useful parameters. This in turn means that calibration shots will have to be performed prior to the diagnostic shots for benchmarking the codes. For instance, exploding pusher capsules that have essentially zero areal density could be used to set a fiducial value for the xenon ratio described above. In addition, the results can be correlated with other diagnostics, such as the neutron time-of-flight measurements (nTOF) for areal density and high resolution x-ray spectroscopy (HRXRS) for hot spot mix.

\section{Neutron-induced reactions}

The first RADCHEM capsules will be doped with ${ }^{124} \mathrm{Xe}$ in order to measure reactions with neutrons produced during the DT-fusion process. ${ }^{124} \mathrm{Xe}$ will undergo both $(n, \gamma){ }^{125} \mathrm{Xe}$ and $(n, 2 n){ }^{123} \mathrm{Xe}$ 
reactions. Noble gases will be collected following a shot using the RAGS apparatus. In this instrument, water vapor, particulates, and reactive gases will first be removed using a series of getters, and the xenon will subsequently be collected on a cryo collection unit for either in-situ gamma spectroscopy or sample removal followed by radiation counting in the Nuclear Chemistry Counting Facility (NCCF). In either case, the ratio of ${ }^{125} \mathrm{Xe} /{ }^{123} \mathrm{Xe}$ will be measured. Since this is a self-tracing reaction, the collection efficiency of both xenon isotopes will be the same and therefore cancel out of the final value.

Several xenon isotopes were considered for this measurement. Originally, ${ }^{134} \mathrm{Xe}(10 \%$ abundant) was used in simulations, but ${ }^{126} \mathrm{Xe}(0.9 \%)$ and ${ }^{124} \mathrm{Xe}(0.9 \%)$ have since been considered. The use of a particular isotope depends on the production cross sections for the neutron reactions of interest and the concentration of background xenon isotopes that could interfere with a measurement. Full-yield ignition shots are expected to use hohlraums that are made from depleted uranium (DU). The 14-MeV neutrons produced in DT-fusion will interact with the DU in the hohlraum, causing it to undergo nuclear fission. Several neutron-rich xenon isotopes are produced as fission fragments. This will create a background of radioactive xenon atoms that will also be collected by the RAGS instrument. In addition, the chamber gas background composition will likely contain some fraction of air, which contains stable xenon isotopes (the results of recent chamber background measurements are being evaluated by C. Velsko). ${ }^{124} \mathrm{Xe}$ produces an isotope ratio that is large enough to be observed over these backgrounds, making it our primary choice of capsule dopant. As seen in Fig. 1, following a DT ignition shot, there will be an array of xenon isotopes present in the chamber from our diagnostic signal, natural air, and fission products. The neutron reaction products of ${ }^{124} \mathrm{Xe}$ are neutron-deficient enough to have a "clean" signal that is free from interfering contaminants. If DU is not used in the hohlraum, this opens up the potential use of other xenon isotopes as capsule dopants. The main issue with ${ }^{124} \mathrm{Xe}$ is that it is only $0.9 \%$ abundant, which means there is a higher cost associated with capsule fabrication. In addition, unused xenon must be recovered and recycled for future capsules, which is not currently part of the capsule fabrication process. If a gold hohlraum is consistently used instead, ${ }^{134} \mathrm{Xe}$ could then be considered. It should be noted that a majority of the initial campaign implosion experiments are expected to use a gold hohlraum, so that RADCHEM using ${ }^{134}$ Xe could diagnose potential capsule failures.

The specific pair of neutron-induced reactions under investigation was chosen on the basis of the energy dependency of their respective cross sections. In Fig. 2 , the $(n, 2 n)$ and $(n, \gamma)$ cross sections are plotted as a function of neutron energy. The $(n, 2 n)$ reaction principally interacts with the primary (14 MeV) neutrons produced in the DT-fusion reaction, whereas the $(n, \gamma)$ reaction is primarily sensitive to neutrons with energy below $10 \mathrm{MeV}$. Although their separate abundances provide information about the neutron energy spectrum, their ratio yields a more easily interpreted value since the effects of the capsule shell's non-uniform spatial distribution and differences in absolute neutron number (yield) are minimized. In Fig. 2 we also compare the simulated cross sections with experimental data. In general, the experimental uncertainties are smallest for low energy neutrons where copious data exists. For intermediate energies, there is less data and evaluations disagree with the data by about $10 \%$. Figure 3 shows an escaped neutron energy spectrum calculated for an equimolar DT capsule fill. The region 
between 3-12 MeV is due to 14-MeV neutrons that have been downscattered in energy as they leave the capsule. The red line shows the same spectrum when the downscattering process is suppressed.

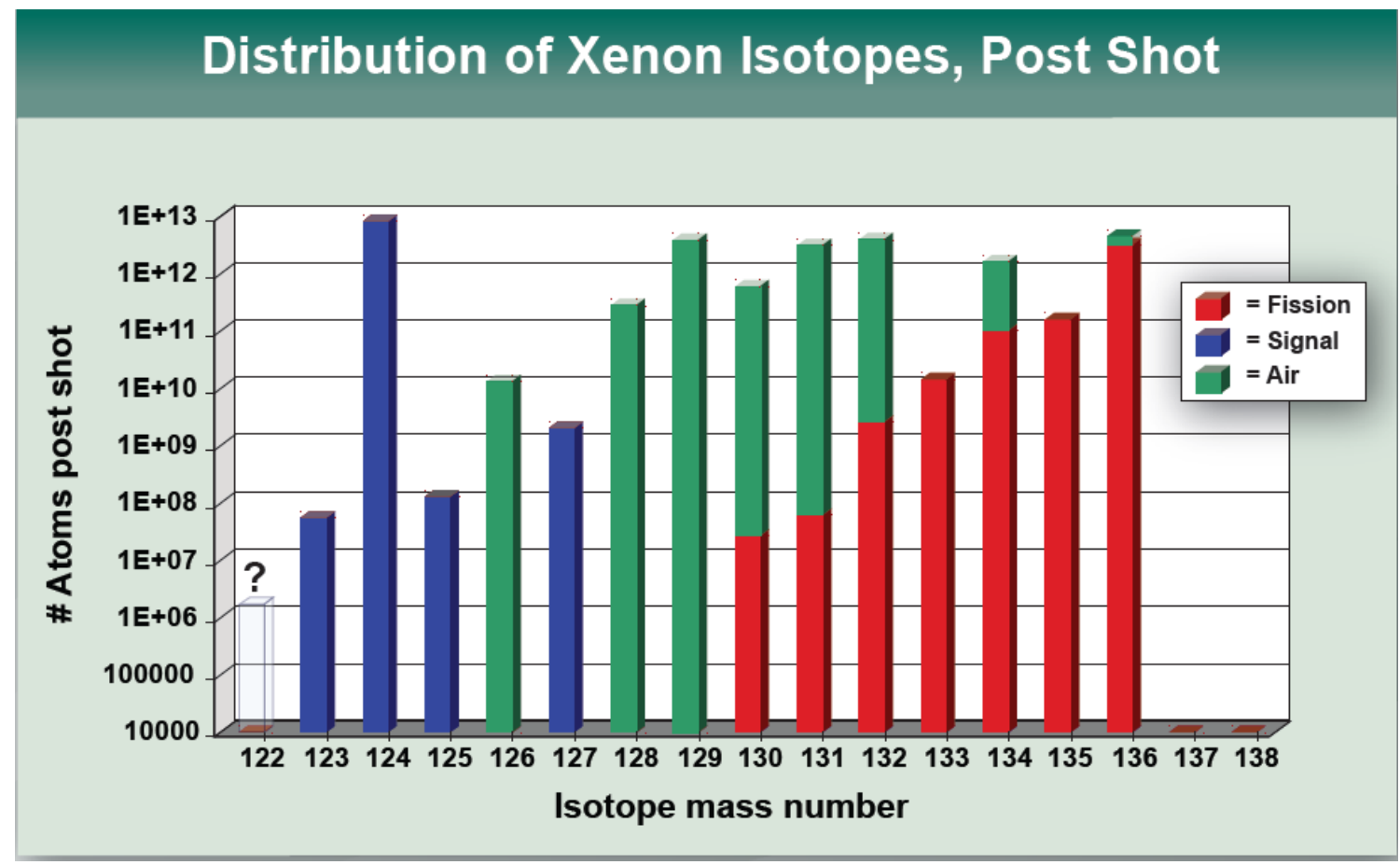

Figure 1. Distribution of xenon isotopes in the NIF chamber following a DT ignition shot. This calculation assumes that $1 \times 10^{13}$ atoms of ${ }^{124} \mathrm{Xe}$ were doped in the capsule ablator shell, the chamber background has the same composition as air, and the hohlraum contains DU. The ${ }^{127} \mathrm{Xe}$ arises from the ${ }^{127} \mathrm{I}(d, 2 n){ }^{127} \mathrm{Xe}$ reaction when ${ }^{127} \mathrm{I}$ is co-loaded in the ablator with the ${ }^{124} \mathrm{Xe}$. Stable isotopes of xenon are ${ }^{124} \mathrm{Xe}$ plus all of the isotopes marked as "air" (in green) in the figure.

Based on these figures the analysis of D-T shots is rather straightforward. The $(n, 2 n)$ signal is caused by $14 \mathrm{MeV}$ neutrons. The $(n, \gamma)$ signal for D-T is caused by down scattered $14-\mathrm{MeV}$ neutrons and thus measures the areal density of the fuel ( $\rho r)$. As the fuel $\rho$ increases, more neutrons will downscatter to lower energy, thereby increasing the $(n, \gamma)$ signal compared to the $(n, 2 n)$ signal, which is only dependent on the DT yield of the capsule. Next we look deeper into this strong correlation between the $(n, \gamma) /(n, 2 n)$ ratio and downscattered neutron fraction. In Fig. 4 we compare the radiochemical signatures with an ideal nTOF that is capable of looking at all angles. This correlation is plotted in Fig. 4 for a set of DT-filled capsule simulations, where the relevant nTOF fraction is the ratio of 6-10 MeV downscattered (DS) neutrons to 12-17 MeV primary (P) neutrons. It should be pointed out that comparisons with nTOF are based upon the so-called $4 \pi$ nTOF. The radiochemical signature is insensitive to possible issues of isotropy in the NIF chamber and provides an independent verification of the reliability of the nTOF-determined fraction. Note that the (125/123) ratio may be generally understood as the ratio of the sum of the low energy, directly produced neutrons plus the lower energy downscattered primary neutrons divided by the primary neutron number: (D + DS)/P. In the limit of negligible downscattered neutron contribution (the so-called zero pr case), the nTOF fraction will vanish 
whereas the (125/123) ratio will not. It is expected that calibration shots using low pr targets would be used to minimize the fixed "background" signal for this series of experiments.

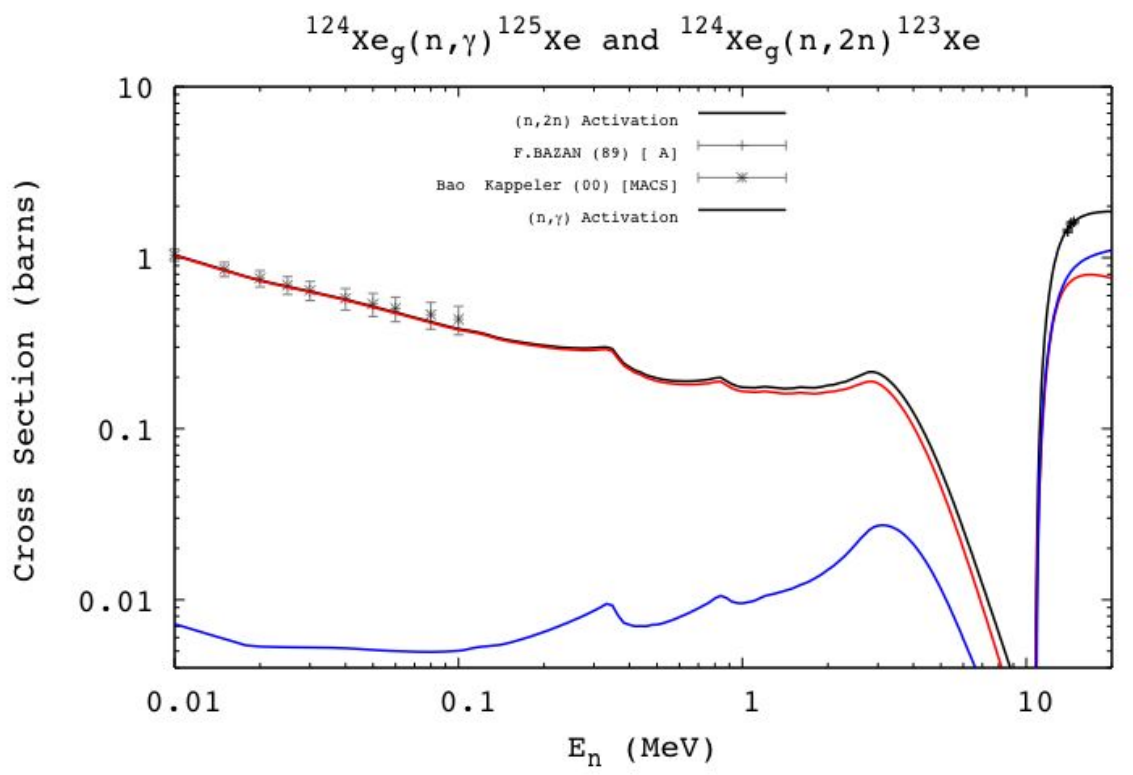

Figure 2. Neutron-induced xenon cross sections as a function of neutron energy. The red curve indicates the amount of activation cross section that proceeds to the ground state, the blue curve is the amount that proceeds to the first isomer, and the black curve gives the total activation cross section. Experimentally derived points are also shown.

As described in Section I above, the results from initial RADCHEM measurements will be xenon isotope ratios. The corresponding downscattered neutron fraction and fuel $\rho$ r will have to be interpreted from this data through the use of explosion code calculations. The zero pr calibrations will be used to set the lower limit on the ${ }^{125} \mathrm{Xe} /{ }^{123} \mathrm{Xe}$ ratio, which will then be correlated with appropriate nTOf measurements. Once capsules with greater than zero pr are used, the codes will have to be used to convert the RADCHEM ratio into a downscattered fraction. 


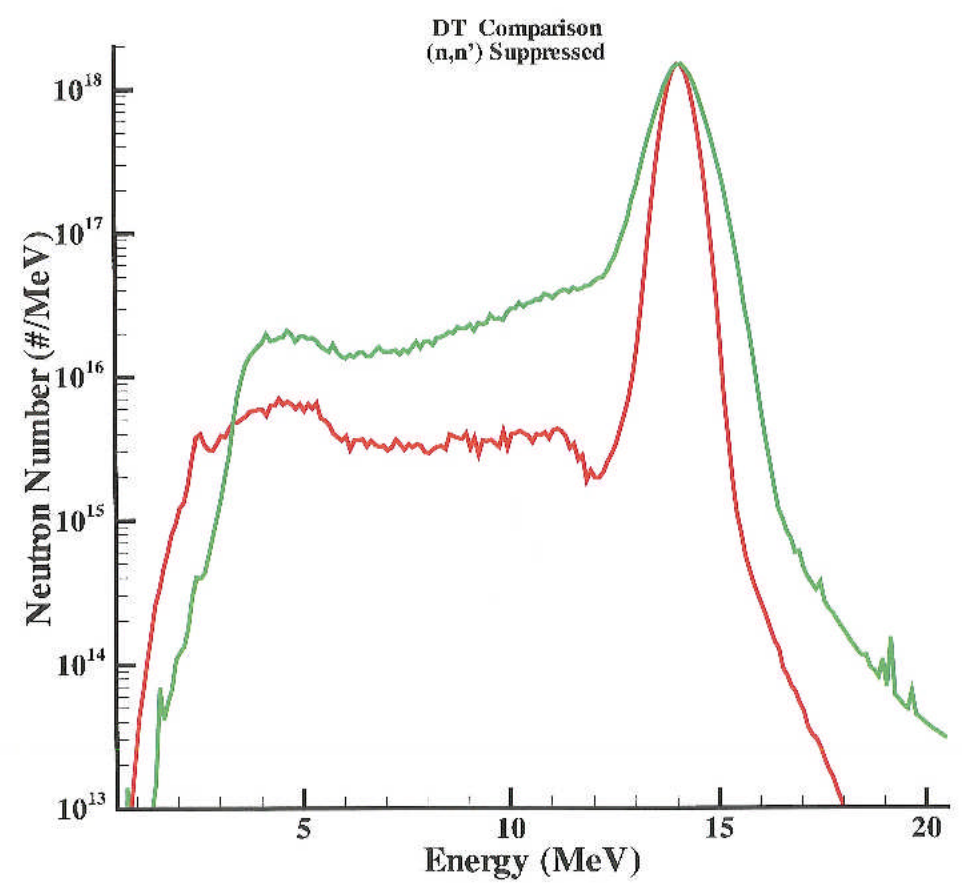

Figure 3. Direct (D) and downscattered (DS) neutron energy spectrum (green line) compared to suppressed downscattered energy spectrum (red line) for an equimolar DT-filled capsule.

\section{Charged particle reactions}

One of the metrics that determines capsule performance is mix. This occurs when part of the ablator shell mixes into the hot, burning fuel, thereby cooling it and reducing the amount of burn that occurs. This in turn affects the overall performance of the capsule, in particular, the yield. Evaluating the extent of the mix that happened during a shot is difficult, especially since the physical process only occurs over a very small physical area of the capsule fuel. Using RADCHEM detectors may be one of the only ways to evaluate the amount of mix that occurred in a capsule. Since the detector atoms will be loaded right at the intersection between the fuel and ablator shell, short-ranged nuclear reactions could be used to evaluate how much of the shell was actually pushed into the fuel. While neutrons will easily escape the burning hot-spot, charged particles such as deuterons, tritons, and alphas will only have a short range, especially as the $\rho r$ of the fuel increases during implosion. By choosing detector elements that are sensitive to activation by these particles, one could evaluate how much of the hot-spot was mixed in with the ablator and therefore evaluate the amount of mix that occurred. Two mix reactions have thus far been considered - the $(d, 2 n)$ and $(\alpha, n)$ reactions. 


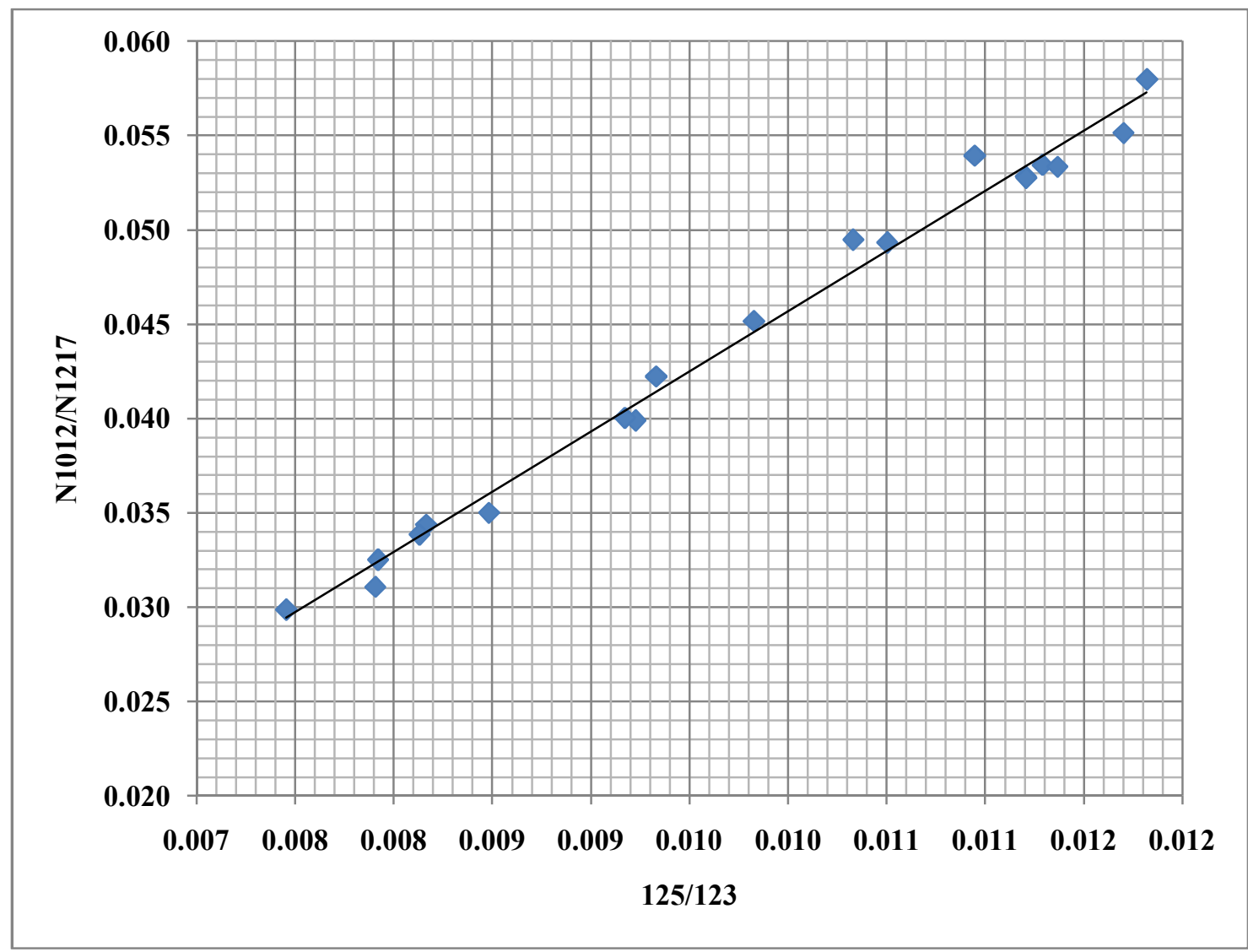

Figure 4. The nTOF-detemined downscattered fraction plotted as a function of $(n, \gamma) /(n, 2 n)$ ratio for DTfilled capsule simulations. Calculations assume that roughly $10^{13}$ atoms of ${ }^{124}$ Xe were loaded into the capsule prior to the shot.

An extended series of simulations were performed that attempted to isolate the effects of two distinct mixing mechanisms expected in NIC point design capsule implosions. These two mix phenomena are typically termed "static" or "dynamic" mix which designates (more appropriately) the way the mix is simulated rather than the underlying physics, which is always "dynamic" in a pristine capsule. Static mix is thought to be induced by rapid introduction of the outer ablator shell material into the gas fill early in the implosion phase, probably caused by the fill tube opening. Recent calculations have indicated that the larger divots or bumps on the surface of a capsule can also induce this type of mix, so it is expected to be ubiquitous in the upcoming implosion campaigns. Calculations that capture this effect faithfully are very time and memory intensive so the usual approximate procedure is to just insert extra ablator mass into the gas fill initially, which is probably a conservative approach as far as the gas-filled region dynamics are concerned but obviously ignores the necessary shell and fuel layer disruption that must have occurred to have the mass enter the gas fill. Dynamic mix is modeled in a slightly more realistic fashion. Shell material is allowed to diffuse across the fuel-shell boundary with a characteristic diffusion length which evolves as the capsule implodes. The simulations performed thus far have used the Rev $4 \mathrm{CH} / \mathrm{Ge}$ capsule design. There has been no systematic study of radiochemical diagnostic signatures of mix effects using the most recent Rev $5 \mathrm{CH} / \mathrm{Ge}$ point design capsule, essentially 
due to the lack of the appropriate simulation capability with HYDRA. This capability is expected to become available by the end of CY10.

\section{A. The $(d, 2 n)$ mix diagnostic}

Protons, deuterons and tritons in the burning region of the capsule may collide with the energetic neutrons produced in the DT-fusion reaction. These particles may acquire a significant fraction of the incident neutrons' energy and subsequently react to release very energetic neutrons in a fusion reaction -- 20-30 MeV neutrons for secondary DT-fusion. If suitable radiochemical tracers are present, these very energetic particles could also react to form products inaccessible by thermal particles. Although these processes will be statistically rare, there might be sufficient signal obtained for failed DT-filled capsules to produce about $1 \times 10^{16}$ neutrons. In these circumstances, about $1 \times 10^{8}{ }^{127} \mathrm{Xe}$ atoms are predicted to form via the ${ }^{127} I(d, 2 n){ }^{127}$ Xe reaction even though the reaction threshold is 4.2 $\mathrm{MeV}$. These energetic deuterons typically have a broad, Gaussian-like distribution in the range $4-12$ $\mathrm{MeV}$ with peak amplitude about $1 \times 10^{-3}$ of the neutron yield. In contrast, the cooler hot spot and lower neutron yields for $\mathrm{TH} / \mathrm{D}(0.02)$ have drastically lower energetic particle production thus mitigating their utility as a hot spot diagnostic.

Two $(d, 2 n)$ reactions have been evaluated using a set of simulations with the mix models described above. The final products are different chemical elements than what was originally loaded in the capsule. This requires the use of a tracer element that is co-located with the detector element of interest. For the ${ }^{127} \mathrm{I}(d, 2 n)^{127} \mathrm{Xe}$ reaction, if the iodine is co-loaded with ${ }^{124} \mathrm{Xe}$ for a simultaneous measurement of the fuel $\rho r$, the resulting ${ }^{125} \mathrm{Xe}$ and ${ }^{123} \mathrm{Xe}$ would act as an indicator of the collection efficiency for the ${ }^{127} \mathrm{Xe}$ produced from ${ }^{127} \mathrm{I}$. The ${ }^{79} \mathrm{Br}(d, 2 n){ }^{79} \mathrm{Kr}$ reaction would require the use of a krypton tracer loaded in the same location as the ${ }^{79} \mathrm{Br}$. Based on an evaluation of the chamber background air and fission product krypton made from $14 \mathrm{MeV}$-neutron induced fission of the DU hohlraum (similar to Fig. 1 above), the most likely candidate for the krypton tracer would be the use of radioactive ${ }^{85} \mathrm{Kr}\left(\mathrm{t}_{1 / 2}=10.8 \mathrm{y}, 100 \% \beta^{-}\right.$decay). Fig. 5 shows the distribution of krypton isotopes expected to be in the NIF chamber following a shot, assuming the background gas has the same composition as air, $5 \times 10^{13}$ atoms of ${ }^{79} \mathrm{Br}$ were loaded into the capsule and ${ }^{85} \mathrm{Kr}\left(10^{13}\right.$ atoms) were used as the tracer material. Being that the results of loading either ${ }^{127} \mathrm{I}$ or ${ }^{79} \mathrm{Br}$ into the capsule are basically the same, loading ${ }^{127}$ I with ${ }^{124} \mathrm{Xe}$ would be the preferred choice as it avoids the requirement of loading a radioactive tracer into the capsule.

\section{B. The $(\alpha, n)$ mix diagnostic}

As described in Section I, alpha particles are the only first-order charged-particle that are directly produced as a result of the DT-fusion process. In addition, alpha particles have a much shorter range than scattered deuterons. As such, they could act as a very sensitive probe to short-range mix processes that occur between the fuel and ablator, or as a result of fill-tube material jetting into the hot spot. The only alpha-induced reaction that results in a gaseous product is the ${ }^{18} \mathrm{O}(\alpha, n)^{21} \mathrm{Ne}$ reaction, as described in Section I. Other potential alpha-induced reactions would all require the use of solid debris collection. 


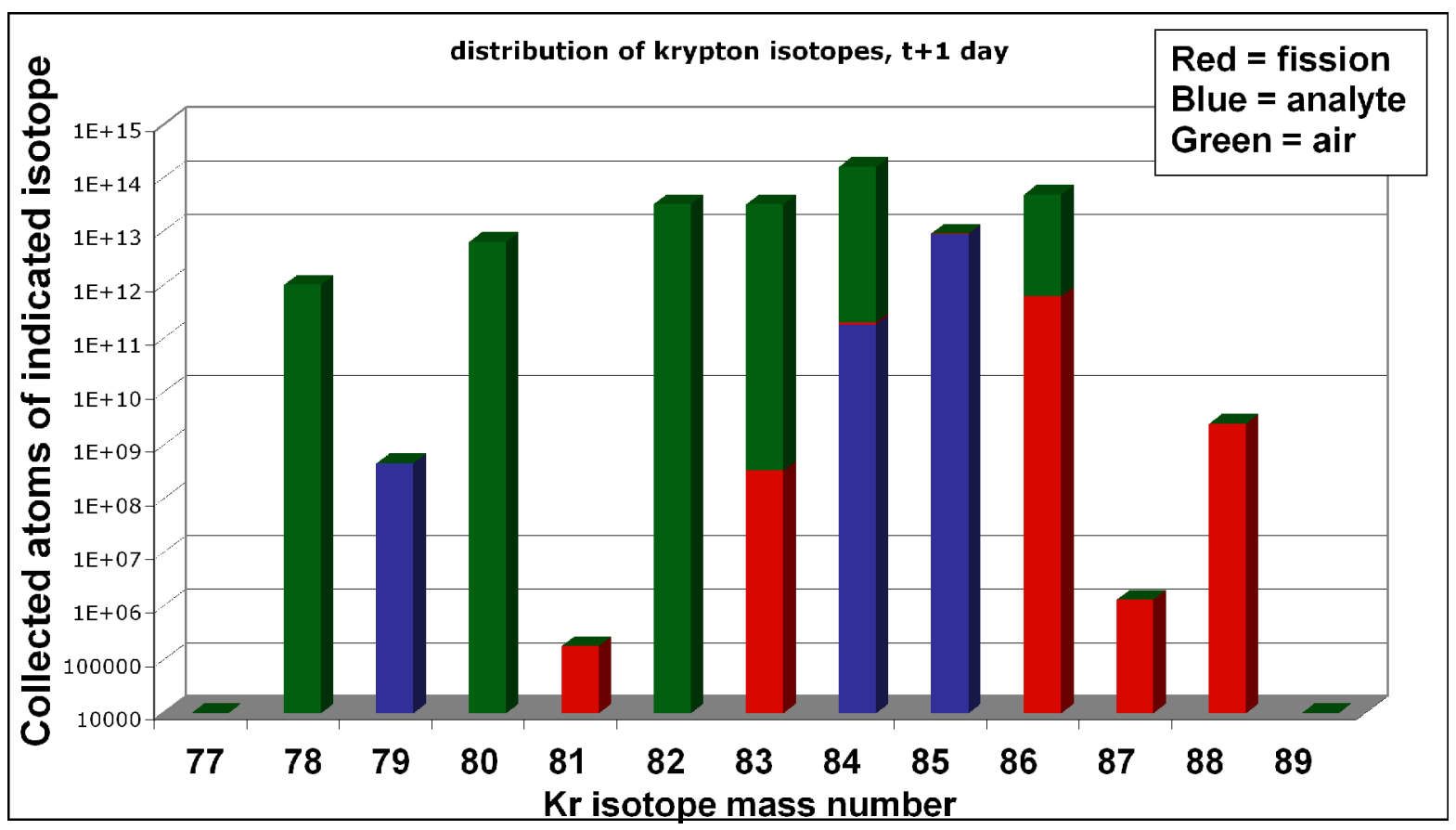

Figure 5. Distribution of krypton isotopes in the NIF chamber following a DT ignition shot. This calculation assumes that $5 \times 10^{13}$ atoms of ${ }^{79} \mathrm{Br}$ and $10^{13}$ atoms of ${ }^{85} \mathrm{Kr}$ tracer were originally doped in the capsule ablator shell, the chamber background has the same composition as air, and the hohlraum contains DU.

Simulations were performed where the location of the doped ${ }^{18} \mathrm{O}$ was varied, starting from the inner-most region of the ablator (closest to the fuel), and moving out until the dopant was farther away. As seen in Fig. 6, the location of the doped material has a significant impact on the number of ${ }^{21} \mathrm{Ne}$ atoms produced in the ${ }^{18} \mathrm{O}(\alpha, n)^{21} \mathrm{Ne}$ reaction. In the same figure, similar results are plotted for a neutron capture reaction $\left({ }^{126} \mathrm{Xe}(n, \gamma){ }^{127} \mathrm{Xe}\right)$. While there is some slight variation as a function of distance, the changes are not as dramatic as those seen in the alphainduced reaction. These simulation results show why alpha particles would be the most sensitive measure of mix. For mix diagnostics, it will be extremely important to evaluate not only how much of a detector element was added to the ablator, but also where it is located. 


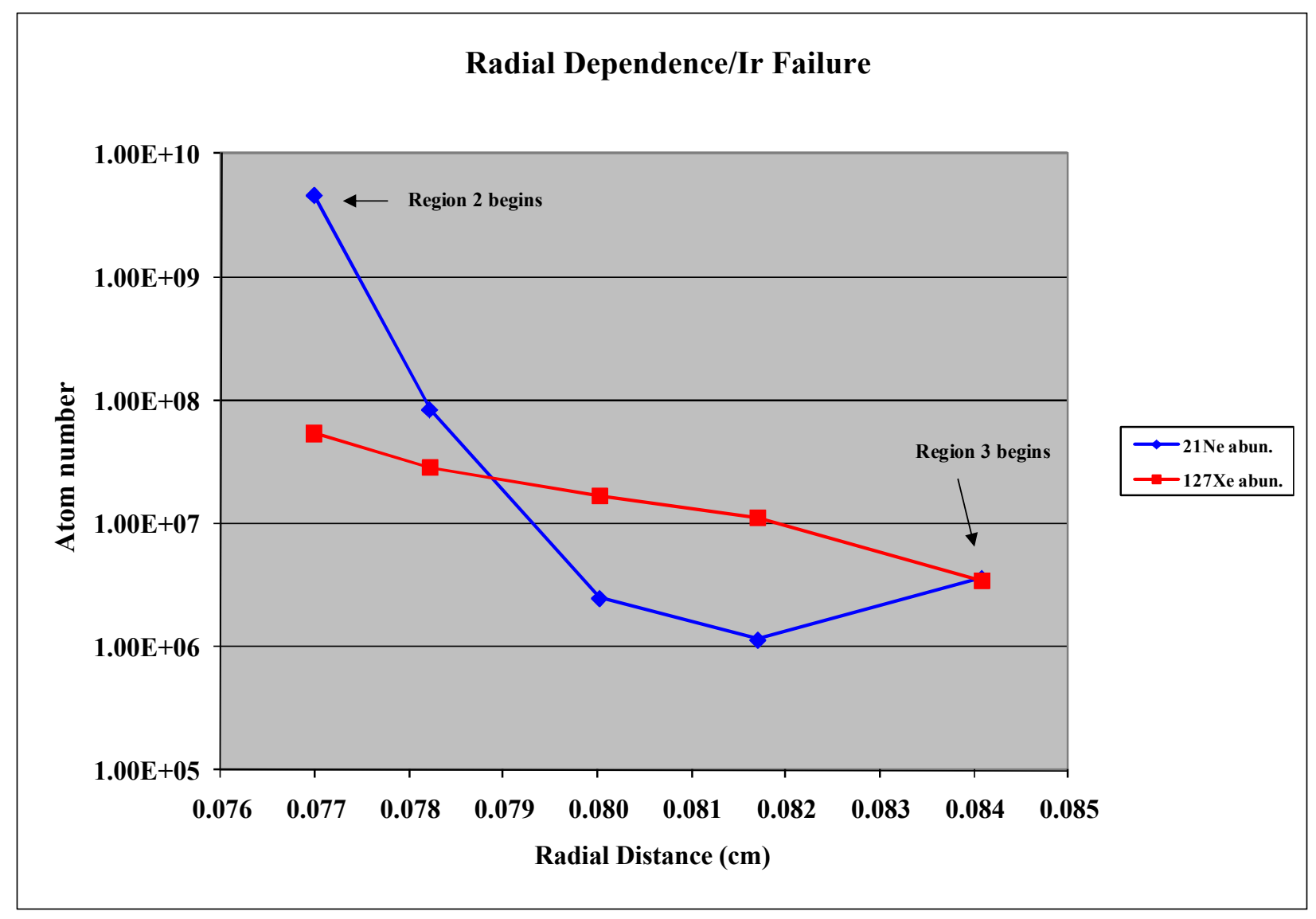

Figure 6. Results from capsule simulations showing the numbers of atoms produced in either the ${ }^{18} \mathrm{O}(\alpha, n){ }^{21} \mathrm{Ne}$ or ${ }^{126} \mathrm{Xe}(n, \gamma){ }^{127} \mathrm{Xe}$ reactions as a function of the location of the doped material from the fuel. Region 2 corresponds to the capsule fuel layer while Region 3 is the innermost ablator fuel layer. The alpha-induced reaction is much more sensitive to the location of the detector element than the deuteron-induced reaction.

\section{The RAGS Apparatus}

The Radiochemical Apparatus for Gaseous Samples (RAGS) for NIF is currently under construction at LLNL and is shown in Fig. 7. The RAGS system should be capable of collecting nearly $100 \%$ of the noble gases produced in an experiment, which arise from reactions of charged-particles with halogens and oxygen or reactions of neutrons with noble gases. The system utilizes the NIF turbopumps to collect a sample of gas from the target chamber following an experiment. The sample gases are then cleaned with a series of SAES chemical getters to remove particulates, water vapor and all nonnoble gases. The cleaned sample is then passed through a series of four identical cryogenic stations with cryo-heads at different temperatures to separate $\mathrm{Xe}, \mathrm{Kr}, \mathrm{Ar}$ and $\mathrm{Ne}$. One of these stations is shown in Fig. 7 and consists of two cryo-heads for further purification of noble gas samples, one for the initial collection of the sample, and a second cryo-head for efficient counting of radioactive noble gases. 


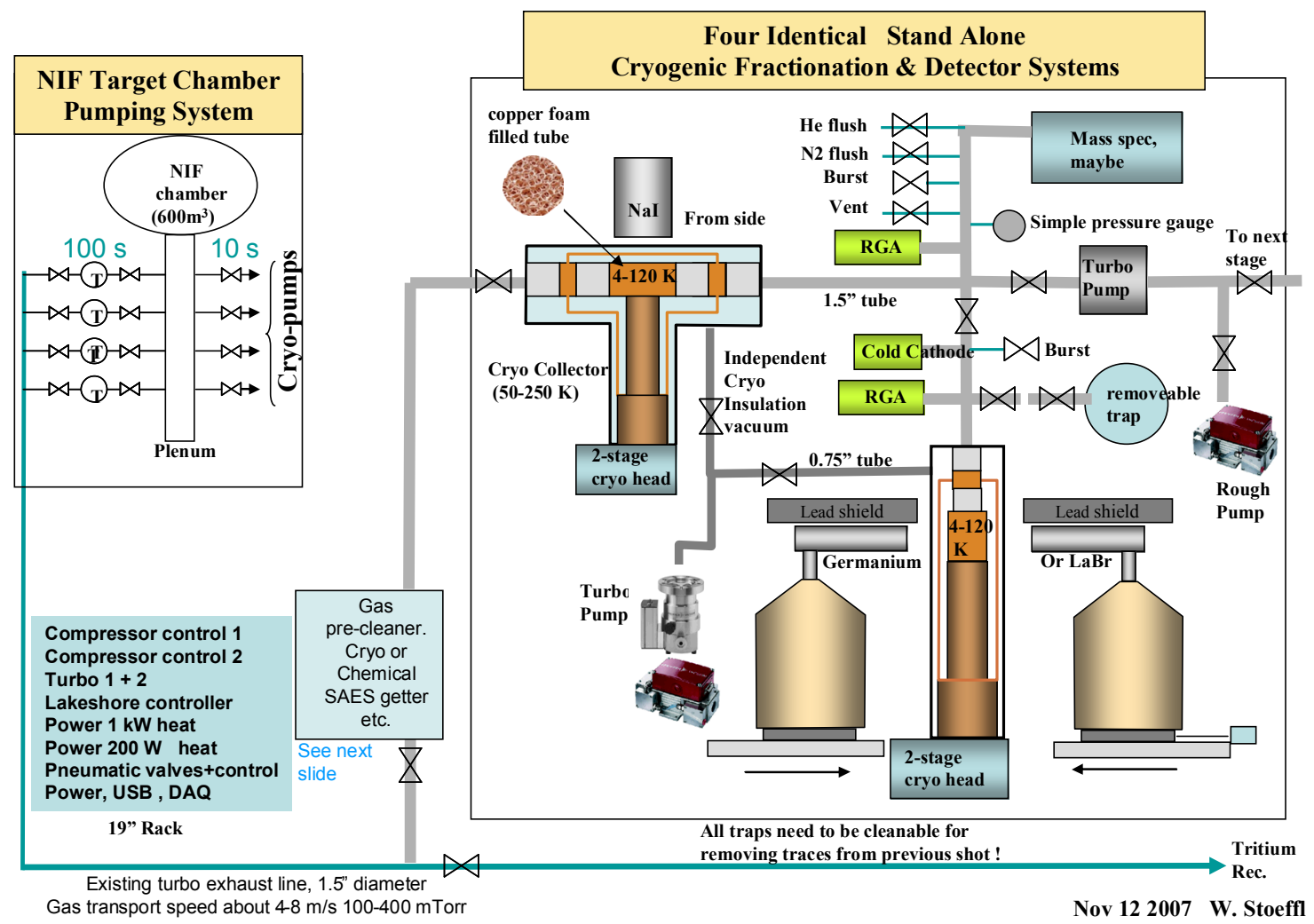

Figure 7. Schematic of the RAGS system. Features include a pre-cleaner system to remove gases other than noble gases, a system of two cryo-heads that have tunable temperatures for flexible collection and purification of the noble gas of interest, and an on-line detection station for preliminary activation determinations.

\section{Future Work}

While the first applications at NIF will focus on noble gas collection and a simplified data analysis, two future developments should greatly add to the value of RADCHEM. The first is the realization that our attempts to reduce the data into separate rhoR and mix diagnostics reduce the information contained in the data. The neutron-induced products are used to measure rhoR but the individual components of the xenon isotope ratio are sensitive by themselves to other things like shape and mix. These other items can be extracted, for example, if correlations with other diagnostics including other RADCHEM data are examined. This effort has been hampered by the necessary code development discussed above but it should be an area of active investigation particularly as data becomes available. The other area of investigation will include advanced collection techniques. Solids and non-radioactive gases offer opportunities for improved diagnostics and open a wider variety of potential capsule dopants and nuclear activations. These will be actively investigated in the future. Materials are currently being studied for their appropriateness to the issue of solid debris collection, and 
recently assigned engineering support is working on the design for an initial debris collection apparatus. Various materials are being fielded in the NIF chamber as ride-along experiments in order to evaluate their behavior during a shot. Debris steering and aerosol assisted collection are also being studied through experiments at other laser facilities. 